\title{
QUALITATIVE THEORY OF PARTIAL DIFFERENCE EQUATIONS (III): FORCED OSCILLATIONS OF PARABOLIC TYPE PARTIAL DIFFERENCE EQUATIONS
}

\author{
SUI SUN CHENG, SHENG-LI XIE AND BING-GEN ZHANG
}

\begin{abstract}
Albstract. Parabolic type partial difference equations with a forcing term is studied in this paper. By means of three averaging techniques, the problem of oscillation of these equations is reduced to that of recurrence relations in one variable. Avariety of oscillation criteria is given for these recurrence relations which in turn yield oscillation criteria for the partial difference equations.
\end{abstract}

\section{Introduction.}

Oscillation properties of functional parabolic equations have been studied by a number of authors (see for example [1, Chapter 6]). In contrast, partial difference equations do not seem to have drawn as much attention. In [2], oscillation criteria have been derived for homogeneous nonlinear partial difference equations of the form

$$
\Delta_{2} u(i, j)=a(j) \Delta_{1}^{2} u(i-1, j)-q(i, j) g(u(i, j-\sigma)), \quad 1 \leq i \leq n, \quad j \geq 0 .
$$

In this paper, we are concerned with forced oscillation of solutions of nonhomogeneous partial difference equations of the form

$$
\Delta_{2} u(i, j)=a(j) \Delta_{1}^{2} u(i-1, j)-p(j) u(i, j-\sigma)+f(i, j), \quad 1 \leq i \leq n, \quad j \geq 0,
$$

where the delay $\sigma$ is a nonnegative integer, $a(j)$ and $p(j)$ are real functions of integral $j$ where $j \geq 0$, and $f(i, j)$ is dependent on integral variables which satisfy $0 \leq i \leq n+1$ and $j \geq 0$. Given a double sequence $\{v(i, j)\}$, the partial difference $\Delta_{2} v(i, j)$ is defined by

$$
\Delta_{2} v(i, j)=v(i, j+1)-v(i, j)
$$

Received May 3, 1993; revised October 15, 1994.

1991 Mathematics Subject Classification. 39A10, 35R10.

Key words and phrases. Partial difference equations, oscillatoin criteria. 
while

$$
\Delta_{1} v(i, j)=v(i+1, j)-v(i, j) .
$$

The usual difference of a sequence $\{w(k)\}$ is defined by

$$
\Delta w(k)=w(k+1)-w(k)
$$

As a consequence,

$$
\Delta_{1}^{2} v(i-1, j)=\Delta_{1}\left(\Delta_{1} v(i-1, j)\right)=v(i+1, j)-2 v(i, j)+v(i-1, j)
$$

A. solution $u(i, j)$ of $(1.1)$ is a double sequence defined for $0 \leq i \leq n+1$ and $j \geq-\sigma$ which satisfies (1.1). Given an arbitrary function $\varphi(i, j)$ defined for $-\sigma \leq j \leq 0$ and $0 \leq i \leq n+1$, and arbitrary functions $g(j)$ and $h(j)$ defined for $j \geq 1$, we can easily show that a unique solution of (1.1) exists and satisfies the conditions

$$
\begin{gathered}
u(0, j)=g(j), \quad j \geq 1 \\
u(n+1, j)=h(j) \quad j \geq 1
\end{gathered}
$$

and

$$
u(i, j)=\varphi(i, j), \quad-\sigma \leq j \leq 0, \quad 0 \leq i \leq n+1 .
$$

Let $w(i, j)$ be a double sequence defined for $0 \leq i \leq n+1$ and $j \geq-\sigma$. Suppose there is some nonnegative integer $T$ such that $w(i, j)>0$ for $1 \leq i \leq n$ and $j \geq T$, then $w(i, j)$ is said to be eventually positive. An eventually negative $w(i, j)$ is similarly defined. The function $w(i, j)$ is said to be oscillatory for $1 \leq i \leq n$ and $j \geq 0$ if it is neither eventually positive nor eventually negative.

\section{Preparatory lemmas}

We shall be concerned with conditions which imply that every solution of (1.1) is oscillatory. For this purpose, we need several preparatory results, the first two of which can be found in [4] and [5] respectively.

Lemma 2.1. Let

$$
g(i, j)=\left\{\begin{array}{ll}
(n-i+1) j /(n+1) & 1 \leq j \leq i \\
(n-j+1) i /(n+1) & i \leq j \leq n
\end{array} .\right.
$$

Then $g(i, j)$ is symmetric, positive for $1 \leq i, j \leq n$ and

$$
\sum_{j=1}^{n} g(i, j) \Delta^{2} v(j-1)=-v(i)+\delta(i), \quad 1 \leq i \leq n
$$


where $\delta(1)=n v(0) /(n+1), \delta(n)=n v(n+1) /(n+1)$ and $\delta(i)=0$ for $2 \leq i \leq n-1$.

Lemma 2.2. The eigenvalue problem

$$
\begin{gathered}
\Delta^{2} w(k-1)+\lambda w(k)=0, \quad 1 \leq k \leq n \\
w(0)=0=w(n+1)
\end{gathered}
$$

is equivalent to the symmetric eigenvalue problem

$$
w(k)=\lambda \sum_{j=1}^{n} g(k, j) w(j), \quad 1 \leq k \leq n
$$

which possesses the positive eigenvalue

$$
\mu=4 \sin ^{2}\left\{\frac{\pi}{2 n+2}\right\} .
$$

This eigenvalue is smaller than all other eigenvalues and its corresponding eigensolution $w(k)$ is given by

$$
w(k)=\left\{\begin{array}{ll}
0 & k=0 \text { or } n+1 \\
\sin (k \pi /(n+1)) & 1 \leq k \leq n
\end{array} .\right.
$$

Note that the reciprocals of the eigenvalue $\mu$ are $0.5,1,1.707,2.618, \ldots$ for $n=$ $1,2,3,4, \ldots$ respectively. These values form a strictly increasing sequence which diverges to infinity.

Lemma. 2.3. Let $\{\omega(i)\}_{0}^{\infty}$ be a sequence and $\omega(i) \neq-1$ for $i \geq 0$. Let

$$
E(k)=\prod_{i=0}^{k-1}(1+w(i)), \quad k \geq 1 .
$$

Then

$$
E(k+1)\left\{\Delta h(k)+\frac{\omega(k)}{1+\omega(k)} h(k)\right\}=\Delta(E(k) h(k)), \quad k \geq 1
$$

Proof. Since

$$
\Delta E(k)=\prod_{i=0}^{k}(1+\omega(i))-\prod_{i=0}^{k-1}(1+\omega(i))=\omega(k) E(k),
$$

thus

$$
\begin{aligned}
& \Delta(E(k) h(k))=h(k) \Delta E(k)+E(k+1) \Delta h(k) \\
= & E(k+1)\left\{\Delta h(k)+\frac{E(k)}{E(k+1)} h(k) \omega(k)\right\}=E(k+1)\left\{\Delta h(k)+\frac{\omega(k)}{1+\omega(k)} h(k)\right\} .
\end{aligned}
$$




\section{Averaging techniques}

There are several averaging techniques which can be used to reduce the problem of oscillation of (1.1) to that of recurrence relations in one variable. First, suppose $u(i, j)$ is a solution of (1.1-1.4), then the function

$$
v(j)=\sum_{i=1}^{n} u(i, j) w(i)
$$

satisfies

$$
\Delta v(j)=a(j) \sum_{i=1}^{n} w(i) \Delta_{1}^{2} u(i-1, j)-p(j) v(j-\sigma)+\sum_{i=1}^{n} f(i, j) w(i) .
$$

for all large $j$. Note that in view of Lemma 2.1,

$$
\begin{aligned}
& \sum_{i=1}^{n} w(i) \Delta_{1}^{2} u(i-1, j)=\sum_{i=1}^{n}\left\{\mu \sum_{s=1}^{n} g(s, i) w(s) \Delta_{1}^{2} u(i-1, j)\right\} \\
= & \mu \sum_{s=1}^{n}\left\{\sum_{i=1}^{n} g(s, i) \Delta_{1}^{2} u(i-1, j)\right\} w(s)=\mu \sum_{s=1}^{n}\left\{-u(s, j)+\delta_{j}(s)\right\} w(s) \\
= & -\mu v(j)+\mu \sum_{s=1}^{n} \delta_{j}(s) w(s)=-\mu v(j)+\delta_{j}(1) w(1)+\delta_{j}(n) w(n) \\
= & -\mu v(j)+\frac{n}{n+1}\{u(0, j) w(1)+u(n+1, j) w(n)\} \\
= & -\mu v(j)+\frac{n}{n+1}(g(j)+h(j) \sin (\pi /(n+1)) .
\end{aligned}
$$

Thus from (3.2), we have

$$
\Delta v(j)+\mu a(j) v(j)+p(j) v(j-\sigma)=\frac{n}{n+1} a(j)(g(j)+h(j)) \sin \frac{\pi}{n+1}+\sum_{i=1}^{n} f(i, j) w(i) .
$$

We summarize these as follows.

Theorem 3.1. If (1.1-1.4) has an eventually positive (or negative) solution, then the following equation,

$$
\Delta v(j)+\mu a(j) v(j)+p(j) v(j-\sigma)=R(j),
$$

also has an eventually positive (respectively negative) solution, where

$$
R(j)=\frac{n}{n+1} a(j)(g(j)+h(j)) \sin \frac{\pi}{n+1}+\sum_{i=1}^{n} f(i, j) w(i), \quad j \geq 0,
$$


Note that, in view of Lemma 2.1,

$$
\sum_{i=1}^{n} g(t, i) \Delta_{1}^{2} u(i-1, j)=-u(t, j)+\Gamma(t, j) \leq \Gamma(t, j)
$$

where

$$
\Gamma(1, j)=\frac{n g(j)}{n+1}, \quad \Gamma(n, j)=\frac{n h(j)}{n+1}, \quad \Gamma(t, j)=0 \quad \text { for } \quad 2 \leq t \leq n-1 .
$$

Thus when $a(j) \geq 0$ for all large $j$, we obtain from (3.9) that

$$
\Delta_{2} \sum_{i=1}^{n} g(t, i) u(i, j)+p(j) \sum_{i=1}^{n} g(t, i) u(i, j-\sigma) \leq a(j) \Gamma(t, j)+\sum_{i=1}^{n} g(t, i) f(i, j)
$$

for $1 \leq t \leq n$ and all large $j$. Summing (3.10) from $t=1$ to $t=n$, we then obtain

$$
\Delta X(j)+p(j) X(j-\sigma) \leq \frac{n a(j)(g(j)+h(j))}{n+1}+\sum_{t, i=1}^{n} g(t, i) f(i, j)
$$

for all large $j$, where

$$
X(j)=\sum_{t=1}^{n} \sum_{i=1}^{n} g(t, i) u(i, j), \quad j \geq 0
$$

Theorem 3.3. Suppose $a(j) \geq 0$ for all large $j$. If (1.1-1.4) has an eventually positive solution, then so does the recurrence relation (3.11). If (1.1-1.4) has an eventually negative solution, then so does the following relation

$$
\Delta X(j)+p(j) X(j-\sigma) \geq \frac{n a(j)(g(j)+h(j))}{n+1}+\sum_{t, i=1}^{n} g(t, i) f(i, j)
$$

\section{Recurrence relations in one variable}

Euqation (3.4) is of the form

$$
\Delta x(j)+P(j) x(j)+Q(j) x(j-\sigma)=\psi(j), \quad j \geq 0
$$

equations (3.7) and (3.11) are of the form

$$
\Delta y(j)+P(j) y(j)+Q(j) y(j-\sigma) \leq \psi(j), \quad j \geq 0
$$


while (3.8) and (3.12) are of the form

$$
\Delta y(j)+P(j) y(j)+Q(j) y(j-\sigma) \geq \psi(j), \quad j \geq 0,
$$

where $\sigma$ is a nonnegative integer. In order to show that (1.1-1.4) is oscillatroy, we need appropriate conditions to ensure that (4.1), (4.2) and (4.3) cannot have eventually positive and /or negative solutions.

In this section, we derive several results which are useful in reducing (4.1), (4.2) and (4.3) into simpler forms.

Suppose first that $P(j) \neq 1$ for $j \geq 0$, let

$$
E(k)=\prod_{j=0}^{k-1}(1-P(j))^{-1}
$$

then in view of Lemma $2.3,(4.1)$ can be written as

$$
\Delta(E(j) y(j))+E(j+1) Q(j) y(j-\sigma)=\psi(j) E(j+1)
$$

or

$$
\Delta(E(j) y(j))+\frac{E(j+1) Q(j)}{E(j-\sigma)} E(j-\sigma) y(j-\sigma)=\psi(j) E(j+1)
$$

If we assume further that $P(j)<1$ for all large $j$, and that $\{y(j)\}$ is an eventually positive solution of (4.1), then $E(j) y(j)$ is an eventually positive solution of (4.4). We summarize these as follows.

Lemma 4.1. Suppose $P(j)<1$ for all large $j$. If (4.1) has an eventually positive (or negative) solution, then

$$
\Delta x(j)+Q(j) \prod_{i=j-\sigma}^{j}(1-P(i))^{-1} x(j-\sigma)=\psi(j) \prod_{i=0}^{j-1}(1-P(i))^{-1},
$$

also has an eventually positive (respectively negative) solution.

Lemma 4.2. Suppose (4.1) (or (4.2)) has an eventually positive solution $\{y(j)\}$, then so does

$$
\Delta w(j)+Q(j)\left\{\exp \sum_{i=j-\sigma}^{j} P(i)\right\} w(j-\sigma) \leq \psi(j) \exp \sum_{i=0}^{j} P(i), \quad j \geq 0 .
$$

Proof. If we multiply both sides of $(4.1)$ by $\exp \{P(0)+\cdots+P(j)\}$, then it is not difficult to sce that

$$
\begin{aligned}
& \Delta\left\{y(j) \exp \sum_{i=0}^{j-1} P(i)\right\}-\{1-\exp (-P(j))-P(j)\} y(j) \exp \sum_{i=0}^{j} p(i) \\
& +Q(j)\left\{\exp \sum_{i=j-\sigma}^{j} P(i)\right\} y(j-\sigma) \exp \sum_{i=0}^{j-\sigma-1} P(i) \leq \psi(j) \exp \sum_{i=0}^{j} P(i) .
\end{aligned}
$$


Since $1-\exp (-x)-x \leq 0$ for all real $x$, we obtain (4.6) from (4.7), where

$$
w(j)=y(j) \exp \sum_{i=0}^{j} P(i) .
$$

Lemma 4.3. Suppose $0 \leq P(j)<1$ for al large $j$. If (4.1) (or (4.2)) has an eventually positive solution $\{y(j)\}$, then so does

$$
\Delta w(j)+\frac{Q(j)}{1-P(j)} w(j-\sigma) \leq \frac{\psi(j)}{1-P(j)}, \quad j \geq 0
$$

Proof. We can write (4.1) in the form

$$
(1-P(j)) \Delta y(j)+P(j) y(j+1)+Q(j) y(j-\sigma)=\psi(j)
$$

from which, we obtain

$$
(1-P(j)) \Delta y(j)+Q(j) y(j-\sigma) \leq \psi(j)
$$

Lemma 4.4. Suppose $P(j) \geq 0, \quad Q(j) \geq 0$ and $\psi(j) \leq 0$ for all large $j$. If (4.1) has an eventually positive solution $\{y(j)\}$, then $P(j)<1$ for all large $j$ (and thus (4.5) and (4.8) has eventually positive solutions).

Proof. We write (4.1) in the form

$$
y(j+1)+Q(j) y(j-\sigma)-\psi(j)=(1-P(j)) y(j)
$$

Our assumptions imply $y(j+1)+Q(j) y(j-\sigma)-\psi(j)>0$ so that $1-P(j)>0$ for all large $j$.

\section{Oscillation criteria of recurrence relations in one variable}

We first deal with the recurrence relation

$$
\Delta y(j)+P(j) y(j)+Q(j) y(j-\sigma) \leq \psi(j), \quad j \geq 0
$$

where $\sigma$ is a nonnegative integer.

An elementary result can be derived as follows. Suppose $P(j) \geq 0$ and $Q(j) \geq 0$ for all large $j$. If $\{y(j)\}$ is an eventually positive solution of (5.1), then for all large $j$,

$$
\Delta y(j) \leq \psi(j)-P(j) y(j)-Q(j) y(j-\sigma) \leq \psi(j)
$$


Summing this inequality from $j=N$ to $j=i$, we obtain

$$
y(i+1) \leq y(N)+\sum_{j=N}^{i} \psi(j) .
$$

If the condition

$$
\liminf _{i \rightarrow \infty} \sum_{j=N}^{i} \psi(j)=-\infty
$$

is imposed, a contradiction will be reached since $y(i+1)>0$ for all large $i$.

Theorem 5.1. Suppose $P(j) \geq 0$ and $Q(j) \geq 0$ for all large $j$. Suppose further that (5.2) is satisfied for some integer $N$. Then (5.1) cannot have an eventually positive solution.

In the above result, $P(j)$ is required to be nonnegative. In view of the Lemmas in the previous section, we can relax this assumption as followas. For instance, in view of Lemma 4.1, Lemma 4.2 and Theorem 5.1, we have the following two corollaries.

Corollary 5.1. Suppose $P(j)<1$ for $j \geq 0$ and $Q(j) \geq 0$ for all large $j$. Suppose further that

$$
\liminf _{i \rightarrow \infty} \sum_{j=N}^{i} \psi(j) \prod_{k=0}^{j}(1-P(k))^{-1}=-\infty,
$$

then (5.1) cannot have an eventually positive solution.

Corollary 5.2. Suppose $Q(j) \geq 0$ for all large $j$. Suppose further that

$$
\liminf _{i \rightarrow \infty} \sum_{j=N}^{i} \psi(j) \exp \sum_{k=0}^{j} P(k)=-\infty,
$$

then (5.1) cannot have an eventually positive solution.

A slightly more complicated result than Theorem 5.1 can be derived as follows.

Theorem 5.2. Suppose $P(j) \geq 0$ and $Q(j) \geq 0$ for all large $j$. Suppose

$$
H(j)=\sum_{k=j}^{\infty} \psi(k)<\infty, \quad j \geq 0 .
$$

If

$$
\liminf _{i \rightarrow \infty} \sum_{j=N}^{i}\{\psi(j)-P(j) \max \{-H(j), 0\}-Q(j) \max \{-H(j-\sigma), 0\}\}=-\infty
$$


then (5.1) cannot have an eventually positive solution.

Proof. Suppose to the contrary that $\{y(j)\}$ is an eventually solution of (5.1). Then

$$
\triangle y(j) \leq \psi(j)-P(j) y(j)-Q(j) y(j-\sigma) \leq \psi(j)
$$

for all large $j$. Thus

$$
y(\infty)-y(i) \leq \sum_{j=i}^{\infty} \psi(j)=H(i)
$$

which implies

$$
y(i) \geq \max \{-H(i), 0\}
$$

From (5.1), we then have

$$
\Delta y(j) \leq \psi(j)-P(j) \max \{-H(j), 0\}-Q(j) \max \{-H(j-\sigma), 0\}
$$

for all large $j$. Thus summing the above inequality, we have

$y(i+1)-y(N) \leq \sum_{j=N}^{i}\{\psi(j)-P(j) \max \{-H(j), 0\}-Q(j) \max \{-H(j-\sigma), 0\}\}, \quad i \geq N$,

which is contradictory to our assumption.

Next, suppose there is a sequence $\{\Psi(j)\}$ which has a nonpositive subsequence $\left\{\Psi\left(j_{k}\right)\right\}$ and satisfies $\Delta \Psi(j)=\psi(j)$ for all large $j$. Suppose further that $P(j) \geq 0$ and $Q(j) \geq 0$ for all large $j$. Then

$$
\Delta\{y(j)-\Psi(j)\} \leq-P(j) y(j)-Q(j) y(j-\sigma) \leq 0
$$

for all large $j$. The nonincreasing sequence $\{y(j)-\Psi(j)\}$ cannot be eventually nonpositive, for otherwise

$$
y(j)-\Psi(j) \leq 0
$$

for all large $j$. But then

$$
0<y\left(j_{k}\right) \leq \Psi\left(j_{k}\right) \leq 0,
$$

for large $k$, which is a contradiction. Thus $\{y(j)-\Psi(j)\}$ is eventually positive. This implies

$$
y(j)>\max \{\Psi(j), 0\} \equiv \Psi^{+}(j)
$$

for all large $j$. Furthermore,

$$
\Delta\{y(j)-\Psi(j)\} \leq-P(j) y(j)-Q(j) y(j-\sigma) \leq-P(j) \Psi^{\dot{+}}(j)-Q(j) \Psi^{+}(j-\sigma)
$$


for all large $j$. Summing the above inequality from $j=N$ to $j=i$, we obtain

$$
0>-(y(i+1)-\Psi(i+1)) \geq y(N)-\Psi(N)+\sum_{j=N}^{i} P(j) \Psi^{+}(j)+Q(j) \Psi^{+}(j-\sigma) .
$$

If the condition.

$$
\limsup _{i \rightarrow \infty} \sum_{j=N}^{i} P(j) \max \{\Psi(j), 0\}+Q(j) \max \{\Psi(j-\sigma), 0\}=\infty
$$

is imposed, a contradiction will be reached. We summarize these as follows.

Theorem 5.3. Suppose $P(j) \geq 0$ and $Q(j) \geq 0$ for all large $j$. Suppose there is a sequence $\{\Psi(j)\}$ which has a nonpositive subsequence $\left\{\Psi\left(j_{k}\right)\right\}$ and satisfies $\Delta \Psi(j)=\psi(j)$ for all large $j$. Suppose further that condition (5.4) is satisfied for some integer $N$. Then (5.1) cannot have an eventually positive solution.

Two variants of Theorem 5.3 can be derived. Both of them require additional information about the sequence $\{\Psi(j)\}$.

Theorem 5.4. Suppose $P(j) \geq 0$ and $Q(j) \geq 0$ for all large $j$. Suppose there is a sequence $\{\Psi(j)\}$ which satisfies $\Delta \Psi(j)=\psi(j)$ for all large $j$ and

$$
\liminf _{i \rightarrow \infty} \Psi(i)=-\beta, \quad \beta>0
$$

Suppose further that the following condition

$$
\limsup _{i \rightarrow \infty} \sum_{j=N}^{i} P(j) \max \{\Psi(i)+\beta, 0\}+Q(j) \max \{\Psi(j-\sigma)+\beta, 0\}=\infty
$$

is satisfied for some integer $N$. Then (5.1) cannot have an eventually positive solution.

Proof. In view of (5.5), $\{\Psi(j)\}$ has a nonpositive subsequence. As we have seen in the derivation of Theorem 5.3, the sequence $\{y(j)-\Psi(j)\}$ is eventually positive and nonincreasing. Thus $y(j)-\Psi(j)$ decreases to a nonnegative constant $\alpha$. We assert that $\alpha \geq \beta$. Indeed, for any $\epsilon>0$,

$$
\alpha+\epsilon>y(j)-\Psi(j) \geq \alpha
$$

for all large $j$. Thus

$$
-\Psi(j)<y(j)-\Psi(j)<\alpha+\epsilon
$$


for alll large $j$, which implies

$$
\beta=\limsup _{j \rightarrow \infty}\{-\Psi(j)\} \leq \alpha+\epsilon
$$

as required. As a consequence,

$$
y(j) \geq \Psi(j)+\beta
$$

for all large $j$. This implies

$$
y(j) \geq \max \{\Psi(j)+\beta, 0\}
$$

for all large $j$. The rest of the proof is similar to that of Theorem 5.3

Theorem 5.5. Suppose $P(j) \geq 0$ and $Q(j) \geq 0$ for all large $j$. Suppose there is a sequence $\{\Psi(j)\}$ which has a constant subsequence $\left\{\Psi\left(j_{k}\right)\right\} \equiv\{h\}$ and $\Psi(j) \geq h$ for all large $j$. Suppose further that $\Delta \Psi(j)=\psi(j)$ for all large $j$. If (5.1) has an eventually positive solution $\{y(j)\}$, then so does the following recurrence relation

$$
\Delta w(j)+P(j) w(j)+Q(j) w(j-\sigma) \leq 0, \quad j \geq 0
$$

Proof. Since

$$
\Delta(y(j)-\Psi(j)+h)=\Delta(y(j)-\Psi(j)) \leq-P(j) y(j)-Q(j) y(j-\sigma) \leq 0,
$$

the sequence $\{y(j)-\Psi(j)\}$ is either eventually positive or eventually nonpositive. We assert that the sequence $\{y(j)-\Psi(j)\}$ satisfies

$$
y(j)-\Psi(j)+h>0
$$

for all large $j$. Otherwise, if $y(j)-\Psi(j)+h \leq 0$ for all large $j$, then

$$
0<y\left(j_{k}\right)=y\left(j_{k}\right)-\Psi\left(j_{k}\right)+h \leq 0
$$

for large $k$, which is a contradiction. If we now set

$$
w(j)=y(j)-\Psi(j)+h, \quad j \geq 0
$$

then

$$
\Delta w(j)+P(j) w(j)+Q(j) w(j-\sigma) \leq P(j)\{h-\Psi(j)\}+Q(j)\{h-\Psi(j-\sigma)\} \leq 0
$$

for all large $j$. This completes our proof. 
We remark that corollaries to Theorems 5.2, 5.3, 5.4 and 5.5 similar to Corollaries 5.1 and 5.2 can be derived by means of the results in Section 4. Since no new principles are involved, the details are omitted.

We remark also that in order to apply Theorem 5.5 to specific situations, additional conditions will be needed which ensure that the recurrence relation (5.7) does not have eventually positive solution. Such conditions, however, have been given in [3, Corollary 2 , Corollary 3, Theorem 3], and will not be repeated here.

We now turn our attention to the recurrence relation

$$
\Delta y(j)+P(j) y(j)+Q(j) y(j-\sigma) \geq \psi(j), \quad j \geq 0
$$

and the recurrence relation

$$
\Delta y(j)+P(j) y(j)+Q(j) y(j-\sigma)=\psi(j), \quad j \geq 0
$$

We are concerned with sufficient conditions under which (5.10) cannot have any eventually negative solutions, and also sufficient condition under which (5.11) has oscillatory solutions only.

Since a solution $\{y(j)\}$ of (5.10) is eventually negative if and only if $\{-y(j)\}$ is an eventually positive solution of

$$
\Delta x(j)+P(j) x(j)+Q(j) x(j-\sigma) \leq-\psi(j), \quad j \geq 0 .
$$

In view of Theorem 5.1, we have the following corollary.

Corollary 5.3. Suppose $P(j) \geq 0$ and $Q(j) \geq 0$ for all large $j$. Suppose further that

$$
\limsup _{i \rightarrow \infty} \sum_{j=N}^{i} \psi(j)=\infty
$$

Then (5.10) cannot have an eventually negative solution.

The next four corollaries follow from Theorems 5.2, 5.3, 5.4 and 5.5 respectively.

Corollary 5.4. Suppose $P(j) \geq 0$ and $Q(j) \geq 0$ for all large $j$. Suppose

$$
K(j)=\sum_{k=j}^{\infty} \psi(j)<\infty, \quad j \geq 0
$$

If

$$
\limsup _{i \rightarrow \infty} \sum_{j=N}^{i} \psi(j)+P(j) \max \{K(j), 0\}+Q(j) \max \{K(j-\sigma), 0\}=\infty,
$$

then (5.10) cannot have an eventually negative solution. 
Corollary 5.5. Suupose $P(j) \geq 0$ and $Q(j) \geq 0$ for all large $j$. Suppose there is a sequence $\{\Psi(j)\}$ which has a nonnegative subsequence $\left\{\Psi\left(j_{i}\right)\right\}$ and satisfies $\Delta \Psi(j)=\psi(j)$ for all large $j$. Suppose further that

$$
\limsup _{i \rightarrow \infty} \sum_{j=N}^{i} P(j) \max \{-\Psi(j), 0\}+Q(j) \max \{-\Psi(j-\sigma), 0\}=\infty .
$$

Then (5.10) cannot have an eventally negative solution.

Corollary 5.6. Suppose $P(j) \geq 0$ and $Q(j) \geq 0$ for all large $j$. Suppose there is a sequence $\{\Psi(j)\}$ which satisfies $\Delta \Psi(j)=\psi(j)$ for all large $j$ and

$$
\lim _{j \rightarrow \infty} \sup \Psi(j)=\alpha>0 .
$$

Suppose further that the following condition

$$
\limsup _{i \rightarrow \infty} \sum_{j=N}^{i} P(j) \max \{-\Psi(j)+\alpha, 0\}+Q(j) \max \{-\Psi(j-\sigma)+\alpha, 0\}=\infty
$$

is satisfied for some integer $N$. Then (5.10) cannot have an eventually negative solution.

Corollary 5.7. Suppose $P(j) \geq 0$ and $Q(j) \geq 0$ for all large $j$. Suppose there is a sequence $\{\Psi(j)\}$ which has a constant subsequence $\left\{\Psi\left(j_{i}\right)\right\} \equiv\{q\}$ and $\Psi(j) \leq q$ for all large $j$. Suppose further taht $\Delta \Psi(j)=\psi(j)$ for all large $j$. If (5.10) has an eventually negative solution, then so does the following recurrence relation

$$
\Delta w(j)+P(j) w(j)+Q(j) w(j-\sigma) \geq 0, \quad j \geq 0 .
$$

We remark that dual results of Corollaries 5.1, 5.2 and other possible corollaries to Theorems $5.2-5.5$ can also be derived easily. As an example, we have the following dual statement of Corollary 5.1,

Corollary 5.8. Suppose $P(j)<1$ for $j \geq 0$ and $Q(j) \geq 0$ for all large $j$. Suppose further that

$$
\limsup _{i \rightarrow \infty} \sum_{j=N}^{i} \Psi(j) \prod_{k=0}^{j}(1-P(k))^{-1}=\infty
$$

then (5.10) cannot have an eventually negative solution.

Finally, we remark that oscillation criteria for (5.11) can be derived easily from the previous results since sufficient conditions for (5.1) to have no eventually positive 
solutions together with sufficient conditions for (5.10) to have no eventually negative solutions are sufficient conditions for (5.11) to be oscillatory.

\section{Oscillation criteria for partial difference equations}

According to the results in the previous Sections, it is now easy to obtain oscillation criteria for the partial difference equation (1.1) subject to (1.2-1.4). We illustrate the principle for obtaining such oscillation criteria as follows. If the conditions

(i) $\mu a(j)<1$ and $p(j) \geq 0$ for $j \geq 0$; and

(ii) $\liminf \operatorname{in}_{i \rightarrow \infty} \sum_{j=N}^{i} R(j) \prod_{k=0}^{j}(1-\mu a(k))^{-1}=-\infty$,

are imposed, then in view of Corollary 5.1, the recurrence relation (3.4) cannot have an eventually positive solution. Thus, by Theorem 3.1, the partial difference equation (1.1) subject to (1.2-1.4) cannot have an eventually positive solution. Similarly, by means of the dual (Corollary 5.8) of Corollary 5.1 and Theorem 3.1, if the conditions

(i) $\mu a(j)<1$ and $p(j) \geq 0$ for $j \geq 0$; and

(ii) $\lim \sup _{i \rightarrow \infty} \sum_{j=N}^{i} R(j) \prod_{k=0}^{j}(1-\mu a(k))^{-1}=\infty$

are satisfied, then (1.1-1.4) cannot have an eventually negative solution. The following Theorem is now clear.

Theorem 6.1. Suppose the following conditions hold:

(i) $R(j)=\frac{n}{n+1} a(j)(g(j)+h(j)) \sin \frac{\pi}{n+1}+\sum_{i=1}^{n} f(i, j) \sin \frac{i \pi}{n+1}, \quad j \geq 0$;

(ii) $\mu a(j)<1$ and $p(j) \geq 0$ for $j \geq 0$;

(iii) $\liminf \operatorname{in}_{i \rightarrow \infty} \sum_{j=N}^{i} R(j) \prod_{k=0}^{j}(1-\mu a(k))^{-1}=-\infty$, and

(iv) lim $\sup _{i \rightarrow \infty} \sum_{j=N}^{i} R(j) \prod_{k=0}^{j}(1-\mu a(k))^{-1}=\infty$,

Then every solution of (1.1-1.4) is oscillatory.

As another example, we can derive the following Theorem from Theorem 3.1, Theorem 5.3 and Corollary 5.5 .

Theorem 6.2. Suppose the following conditions hold:

(i) $R(j)=\frac{n}{n+1} a(j)(g(j)+h(j)) \sin \frac{\pi}{n+1}+\sum_{i=1}^{n} f(i, j) \sin \frac{i \pi}{n+1}, \quad j \geq 0$;

(ii) $a(j) \geq 0$ and $p(j) \geq 0$ for $j \geq 0$;

(iii) there is an oscillatory sequence $\{\Psi(j)\}$ such that $\Delta \Psi(j)=\psi(j)$ for $j \geq 0$;

(iv) $\lim \sup _{i \rightarrow \infty} \sum_{j=N}^{i} \mu a(j) \max \{\Psi(j), 0\}+p(j) \max \{\Psi(j-\sigma, 0\}=\infty$; and

(v) $\lim _{i \rightarrow \infty} \sup \sum_{j=N}^{i} \mu a(j) \max \{-\Psi(j), 0\}+p(j) \max \{-\Psi(j-\sigma), 0\}=\infty$.

Then every solution of $(1.1-1.4)$ is oscillatory.

As a final example, we can derive the following result from theorem 3.2 , Theorem 5.4 and Corollary 5.7. 
Theorem 6.3. Suppose the following conditions hold:

(i) $a(j) \geq 0$ and $p(j) \geq 0$ for $j \geq 0$;

(ii) $\psi(j)=a(j)\{h(j)+g(j)\}+\sum_{i=1}^{n} f(i, j), \quad j \geq 0$;

(iii) there is a sequence $\{\Psi(j)\}$ such that $\Delta \Psi(j)=\psi(j)$ for $j \geq 0$;

(iv) $\{\Psi(j)\}$ has constant subsequences $\left\{h_{1}\right\}$ and $\left\{h_{2}\right\}$, and $h_{1} \leq \Psi(j) \leq h_{2}$ for $j \geq 0$;

(v) every solution of the equation

$$
\Delta w(j)+p(j) w(j-\sigma)=0, \quad j \geq 0
$$

is oscillatory. Then every solution of (1.1-1.4) is oscillatory.

There are many other combinations, the principle, however, is the same.

\section{Acknowledgement}

The first author is supported by th NSC of R.O.C. and the third author is partially supported by NNSF of China.

\section{References}

[1] D. D. Bainov and D. P. Mishev, Oscillation Theory for Neutral Differential Equations with Delay, Adam Hilger, Bristol, 1991.

[2] S. S. Cheng and B. G. Zhang, "Qualitiative theory of partial difference equations (I): Oscillation of nonlinear partial difference equations," Tamkang J. Math., 25 (1994), 279-288.

[3] S. S. Cheng, S. L. Xie and B. G. Zhang, "Qualitative theory of partial difference equations (II): Oscillation criteria, for direct control systems in several variables," Tamkang J. Math., Vol.25, no.1, 1995.

[4] S. S. Cheng, "A discrete analogue of the inequality of Lyapunov," Hokkaido Math. J., 12 (1983), 105-112.

[5] S. S. Cheng, "Discrete quadratic Wirtinger's inequalities," Linear Alg. Appl., 94 (1987), 57-73.

[6] N. Yoshida, "Oscillation of nonlinear parabolic equations with functional arguments," Hiroshima Math. J., 16 (1986), 305-314.

Department of Mathematics, Tsing Hua University, Hsinchu, Taiwan, 300043 R.O.C.

Department of Mathematics, Jinzhou Teacher's College, Hubei, China, 434100.

Department of Mathematics, Ocean University of Qingdao, Qingdao, China, 266003. 